\title{
Characteristic and Management of Symptomatic Septum Pellucidum Cyst in Extreme Elderly Patient: Case Report and Literature Review
}

\author{
Naokado IKedA, ${ }^{1}$ Yoji Tamura, ${ }^{1}$ Yoko Matsushita, ${ }^{2}$ Takuya Kanemitsu, ${ }^{1}$ \\ Naosuke Nonoguchi, ${ }^{1}$ Motomasa Furuse, ${ }^{1}$ Shinji Kawabata, ${ }^{1}$ Toshihiro Takami, ${ }^{1}$ \\ Toshihiko KUROIWA, ${ }^{1,2}$ and Masahiko WANIBUCHI ${ }^{1}$
}

${ }^{1}$ Department of Neurosurgery and Endovascular Neurosurgery, Osaka Medical and Pharmaceutical University, Takatsuki, Osaka, Japan

${ }^{2}$ Department of Neurosurgery, Tesseikai Neurosurgical Hospital, Shijonawate, Osaka, Japan

\begin{abstract}
Septum pellucidum cyst is rare and is defined as a fluid-filled space between the lateral ventricles; it has a width of $10 \mathrm{~mm}$ or more. In this case report, a surgical patient of symptomatic septum pellucidum cyst (SPC) in extreme age is described. To the best our knowledge, this is the first report of an extremely aged patient with symptomatic SPC that was successfully treated using a flexible neuroendoscope. An 85-year-old male complained of gradually worsening gait disturbance, dementia, and urinary incontinence without headache and was admitted to our hospital. MRI revealed a huge cyst between the lateral ventricles as well as ventricle dilatation with periventricular hyperintensity in T2weighted image. The patient was diagnosed with symptomatic hydrocephalus with SPC and underwent neuroendoscopic fenestration of the cyst with the use of a flexible endoscope without cerebrospinal fluid shunt placement. Immediately after the surgery, the patient's gait disturbance and dementia were dramatically improved. In extremely aged patients, SPC tended to develop with idiopathic normal pressure hydrocephalus-like symptoms, including gait disturbance without increasing intracranial pressure, sensorimotor disturbances, and psychological disorders. Neuroendoscopic cyst fenestration with the use of a flexible scope for SPC is a less-invasive procedure and should be considered even for extreme elderly symptomatic patients.
\end{abstract}

Keywords: elderly patient, endoscopic fenestration, flexible endoscope, hydrocephalus, septum pellucidum cyst

\section{Introduction}

Cavum septum pellucidum is a normal persistent cavity between the two leaflets of the septum pellucidum. This is common among newborns and generally disappears during infancy as the two leaflets of the septum unite. ${ }^{1)}$ However, approximately $15 \%$ of those may still found in the adult. In the adult population, the findings are associated with repetitive head injuries and schizophrenia. ${ }^{2}$ Septum pellucidum cyst (SPC) is characterized by a noncommunicating, fluid-filled space with a width of greater than $10 \mathrm{~mm}$ and a lateral bowing of the septal walls of the cavum septum pellucidum; it is observed in $0.04 \%$ of the population. ${ }^{3-5)}$ Occasionally, SPCs become symptomatic and increase intracranial pressure and sensorimotor disturbances as well as induce psychotic or autonomic disorders. Herein, we present a rare case of successfully treated extremely aged symptomatic SPC and discuss the characteristic of the pathology and therapeutic strategy in elderly patients.

\section{Case Report}

An 85-year-old male presented to the outpatient service of our department with gradually worsening gait distur-

Received October 23, 2021; Accepted December 20, 2021

Copyright (C) 2022 The Japan Neurosurgical Society

This work is licensed under a Creative Commons Attribution-NonCommercial-NoDerivatives International License. 


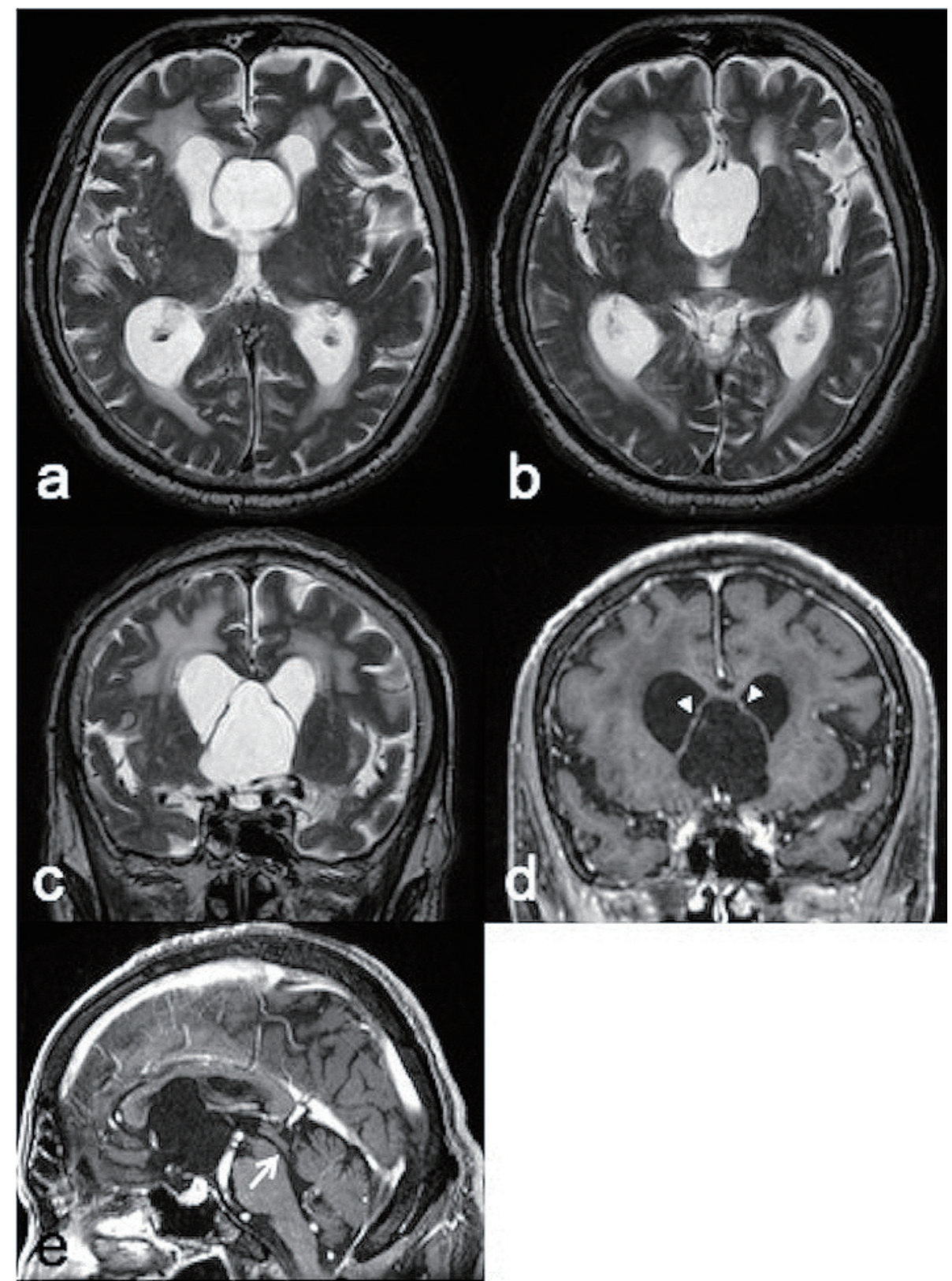

Fig. 1 Magnetic resonance images before the operation demonstrated a cystic lesion between the bilateral anterior horns of the lateral ventricles with periventricle hyperintensity in T2-weighted image (a, b, c). T1-weighted coronal image with gadolinium administration revealed the bilateral septal veins (arrowhead) located lateral to the cyst (d). The cyst wall expanded to the corpus callosum and the floor of the third ventricle. The aqueduct (arrow) was not obstructed by the cyst (e).

bance for 2 years. Six months before the admission, he was diagnosed with lumbar spinal canal stenosis, and lumbar bilateral fenestration had been performed. After the surgery, the numbness of his bilateral lower limbs improved, but his gait disturbance worsened. He denied experiencing headache, nausea, or blurred vision, which suggested intracranial hypertension. Neurological examination revealed that the patient suffered mild cognitive impairment (Mini-Mental State Examination (MMSE) score of 21/ 30), but he did not experience weakness, sensory disturbance, or cerebellar ataxia in his four extremities. How- ever, his gait was very unstable with wide based short steps and the body bent forward. The patient also suffered from frequent urination and urinary incontinence. MRI revealed a cyst containing cerebrospinal fluid (CSF) between the bilateral mildly dilated lateral ventricles. The T2weighted image revealed periventricle white matter hyperintensity. The cyst wall was not enhanced with Gadolinium-DTPA administration. The cyst occupied the anterior part of the third ventricle and extended to the height of the corpus callosum (Fig. 1). The bilateral foramen of Monro was not easily detected, but the aqueduct 


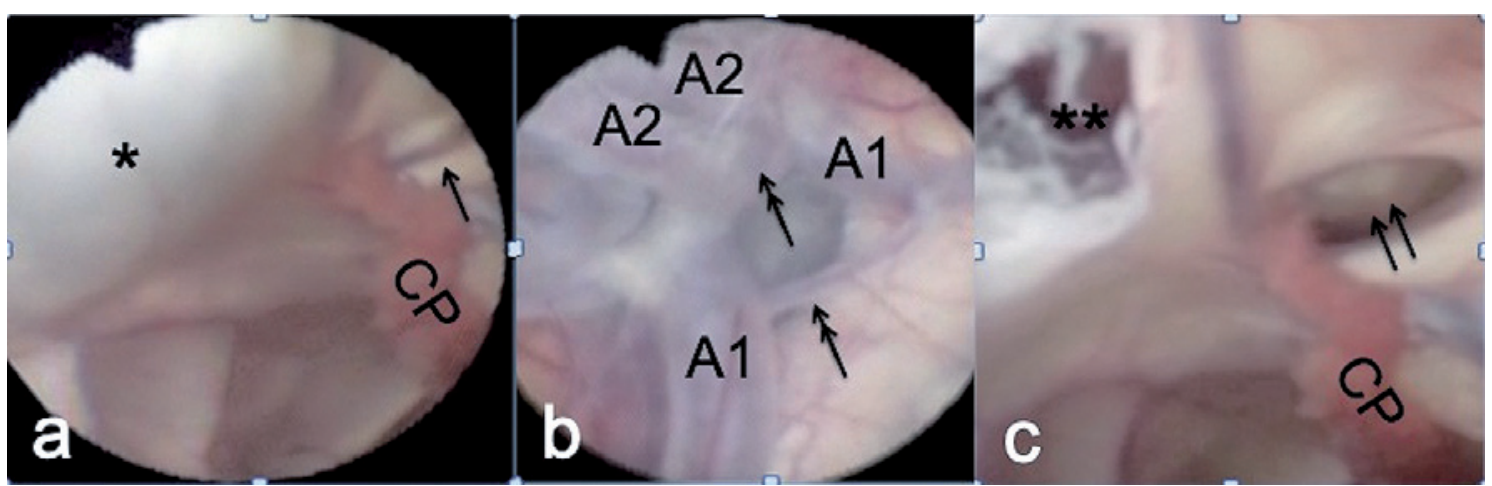

Fig. 2. Intraoperative photographs during neuroendoscopic fenestration of the septum pellucidum cyst.

a: The medial wall of the right lateral ventricle (asterisk) was expanded with lateral bowing by the septum pellucidum cyst, and the right foramen of Monro exhibited a slit-like characteristic (arrow).

b: After fenestration of the cyst wall, the anterior cerebral arteries, duplicated anterior communicating arteries (harpoon), and their perforators were observed through the dilated wall of the cavum septum pellucidum.

c: The stoma-fenestrated right lateral ventricle and the septum pellucidum lamina (double asterisk) were patent, and the right foramen of Monro (double arrow) dilated after the neuroendoscopic fenestration.

A1: anterior cerebral artery horizontal potion, A2: anterior cerebral artery vertical potion.

CP: choroid plexus of the right lateral ventricle.

was not obstructed, and the third and fourth ventricle was only mildly dilated in the images.

Due to the location and the fluid in the cyst, the patient was diagnosed with normal pressure hydrocephalus associated with a huge SPC. Neuroendoscopic fenestration of the SPC was performed via the right anterior horn of the lateral ventricle with the use of a flexible endoscope. The initial pressure after the ventricular tap was normal. The right foramen of Monro exhibited a slit-like characteristic but was not obstructed, and the medial wall of the right lateral ventricle was lateralized (Fig. 2A). The lateralized medial wall of the lateral ventricle and the lamina of the septum pellucidum was fenestrated using a monopolar coagulator and dilation with a balloon catheter. After the endoscope was inserted to the cyst through the fenestrated stoma, the anterior communicating artery complex and its perforators were observed through the lamina of the septum pellucidum (Fig. 2B). Another stoma was fenestrated in the lamina of the septum pellucidum at just above the tuber cinereum. After making the two stomas, the septum pellucidum wall pulsated, and the right foramen of Monro dilated (Fig. 2C). A day after the operation, the patient could stably walk, and MMSE, which was performed 7 days after surgery, revealed improvement in cognitive function (MMSE score of 30/30). Frequent urination and urinary incontinence also gradually improved 1 month after the operation. MRI, which was performed 6 months after the surgery, revealed that the size of the lateral ventricle normalized and that the area of hyperintensity around the lateral ventricles diminished in the T2-weighted image. A flow void was observed near the stoma of the septum pellucidum in the T2-weighted image (Fig. 3). The patient's symptom did not recur for 3 years after surgery, and he

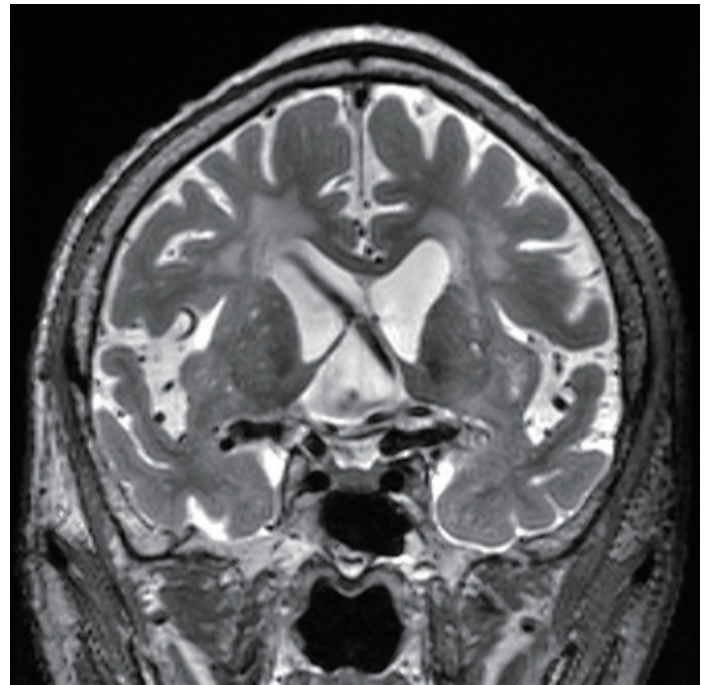

Fig. 3 Coronal magnetic resonance image 6 months after surgery demonstrated flow void near the stoma in the T2weighted image. The size of the lateral ventricles was normalized, and the periventricular hyperintensity area diminished.

was able to walk by himself without assistance.

\section{Discussion}

Generally, symptomatic SPCs occur in children. To the best of our knowledge, this is the first case to report an extremely aged patient with symptomatic SPC that was successfully treated with cyst fenestration using a flexible neuroendoscope. Generally, symptomatic SPCs develop with intracranial hypertension-related symptoms. The most commonly presenting symptoms were intermittent head- 
Table 1 The clinical presentation in the reported cases of septum pellucidum cyst aged above 50 years old

\begin{tabular}{lcccccc}
\hline $\begin{array}{c}\text { Author and } \\
\text { Published year }\end{array}$ & Age & Sex & $\begin{array}{c}\text { Previous } \\
\text { head injury }\end{array}$ & Headache & $\begin{array}{c}\text { Cognitive } \\
\text { dysfunction, dementia }\end{array}$ & $\begin{array}{c}\text { Gait } \\
\text { disturbance }\end{array}$ \\
\hline Love et al. ${ }^{12)}$ 1956 & 61 & $\mathrm{~F}$ & $\mathrm{nd}$ & $\mathrm{y}$ & $\mathrm{n}$ & $\mathrm{n}$ \\
Silbert et al. $^{6}$ 1993 & 61 & $\mathrm{M}$ & $\mathrm{y}$ & $\mathrm{y}$ & $\mathrm{y}$ & $\mathrm{y}$ \\
& 60 & $\mathrm{~F}$ & $\mathrm{n}$ & $\mathrm{y}$ & $\mathrm{n}$ & $\mathrm{n}$ \\
Meng et al. ${ }^{13)}$ 2006 & 60 & $\mathrm{M}$ & $\mathrm{nd}$ & $\mathrm{y}$ & $\mathrm{n}$ & $\mathrm{y}$ \\
Chen LM et al. ${ }^{14)} 2017$ & 58 & $\mathrm{M}$ & $\mathrm{n}$ & $\mathrm{y}$ & $\mathrm{y}$ & $\mathrm{y}$ \\
Present case 2021 & 85 & $\mathrm{M}$ & $\mathrm{n}$ & $\mathrm{n}$ & $\mathrm{y}$ & \\
\hline
\end{tabular}

nd; not described

ache and unconsciousness, probably due to a sudden transient obstruction of the foramen of Monro. Occasionally, they develop with psychotic or autonomous disorder caused by compression to the limbic system or hypothalamic structure. ${ }^{6.7)}$ The preoperative symptoms of the case presented are suggestive of idiopathic normal pressure hydrocephalus, including gait disturbance. The reported elderly cases of symptomatic SPCs, including the case described here, are shown in Table $1^{6,12-14)}$ Majority of the patients aged above 50 years complained of gait disturbance (about 67\%). On the other hand, the reported cases, except the case presented here, complained of headache that might be related to high intracranial pressure. In more extreme elderly patients, similar to the case presented here, even with SPCs, normal pressure hydrocephalus-like symptom was first appeared before raising intracranial pressure. We speculated that depend on decreased brain volume compared with younger age.

In the presented case, the more significant dilatation of the bilateral lateral ventricles compared with the third and fourth ventricle. Additionally, the intraoperative endoscopic findings (slit-like narrowing of the right foramen of Monro) indicated that incomplete obstruction of the foramen of Monro by the expanded SPC. These was thought to be caused developed symptoms. The mechanisms for the expansion of SPC are completely unknown. The check-valve phenomenon of slit formation in the bottom wall of the cyst and CSF production from the protruded choroid plexus of the third ventricle into the cyst is speculated to be one of the mechanisms for the cyst expansion. ${ }^{8}$ Furthermore, head trauma is thought to be one of the triggers of these changes in the cavum septum pellucidum and another midline congenital cyst. ${ }^{8}{ }^{8}$ However, in our literature review of the elderly patient population, only one of six patients with symptomatic SPCs had a history of head trauma. Accordingly, it remains peculiar especially in the elderly population because the cyst perhaps asymptomatically exists for an extremely long time.

Since Jackowski et al. reported the first endoscopic fen- estration of cyst in 1995, ${ }^{9)}$ this procedure has become the first choice for symptomatic SPCs. The most common approach involves ventricular tap through the anterior horn of the right lateral ventricle and penetration of the septum pellucidum leaflets to the ipsilateral ventricle. ${ }^{10)}$ In younger patients with symptomatic SPCs, whose ventricles are not remarkably dilated, the anterior horn of the lateral ventricle is often difficult to access using the endoscope. It is also difficult to penetrate the leaves of the septum pellucidum due to perpendicular trajectory for the penetration, especially when using rigid endoscopes. While Simon et al. proposed the technique of direct penetration through the interforniceal approach using the rigid scope and navigation system guidance to resolve these potential problems, long-term prognosis was not proven. ${ }^{10)}$ In majority of the reported surgical SPCs, the rigid endoscope was used. The limitation of the use of the rigid scope is operative field obtained by the single trajectory. On the other hand, the flexible neuroendoscope allowed a safer fenestration to the perpendicular structures to the approach axis and to multiple sites as our presented case if needed. ${ }^{11)}$ In elderly symptomatic patients with SPCs, for their tolerance to ventricle dilatation and CSF collection, the ventricle dilatation is usually co-existed and sometimes remarkable. Thus, ordinal approach and penetration are easier and safer in young patients than in elderly patients, especially when using a flexible endoscope. Even in extreme elderly patients, neuroendoscopic penetration should be strongly considered if the general condition is tolerable for general anesthesia.

\section{Conclusion}

Symptomatic SPCs in elderly patients tend to develop with only normal pressure hydrocephalus-like symptoms, without symptoms that are related to increased intracranial pressure. The incidence of symptomatic SPC in extreme elderly patients, like the case presented here, is expected to increase with the aging society. Neuroendoscopic 
cyst fenestration should be considered as a safe and convenient therapeutic procedure even for extreme elderly patients.

\section{Acknowledgments}

The authors would like to thank Enago (www.enago.jp) for the English language review.

\section{Conflicts of Interest Disclosure}

All authors have no conflict of interest.

\section{References}

1) Shaw CM, Alvord EC: Cava septi pellucidi et vergae: their normal and pathogical states. Brain 92: 213-223, 1969

2) Lancon JA, Haines DE, Raila FA, Parent AD, Vedanarayanan VV: Expanding cyst of the septum pellucidum. Case report. J Neurosurg 85: 1127-1134, 1996

3) Sarwar M: The septum pellucidum: normal and abnormal. AJNR Am J Neuroradiol 10: 989-1005, 1989

4) Sayama CM, Harnsberger HR, Couldwell WT: Spontaneous regression of a cystic cavum septum pellucidum. Acta Neurochir 148: 1209-1211, 2006

5) Wang KC, Fuh JL, Lirng JF, Huang WC, Wang SJ: Headache profiles in patients with a dilatated cyst of the cavum septi pellucidi. Cephalalgia 24: 867-874, 2004

6) Silbert PL, Gubbay SS, Vaughan RJ: Cavum septum pellucidum and obstructive hydrocephalus. J Neurol Neurosurg Psychiatry 56: 820-822, 1993

7) Amin BH: Symptomatic cyst of the septum pellucidum. Childs
Nerv Syst 2: 320-322, 1986

8) Sagan L, Limanowka B, Herbowski L, Poncyljusz W, Gizewska M: Expanding cyst of the septum pellucidum - endoscopic observations on the mechanism of development and results of treatment. Neurol Med Chir 60: 202-208, 2020

9) Jackowski A, Kulshresta M, Sgouros S: Laser-assisted flexible endoscopic fenestration of giant cyst of the septum pellucidum. $\mathrm{Br}$ J Neurosurg 9: 527-531, 1995

10) Simonin A, Bangash O, Chandran AS, Uvelius E, Lind C: Endoscopic fenestration of cavum septum pellucidum cysts to the third ventricle: Technical Note. Oper Neurosurg 19: E434-E439, 2020

11) Nishijima $Y$, Fujimura M, Nagamatsu K, Kohama M, Tominaga T: Neuroendoscopic management of symptomatic septum pellucidum cavum vergae cyst using a high-definition flexible endoscopic system. Neurol Med Chir 49: 549-552, 2009

12) Love JG, Hollenhorst RW: Bilateral palsy of the sixth cranial nerve caused by a cyst of the septum pellucidum (fifth ventricle) and cured by pneumoencephalography. Proc Staff Meet Mayo Clin 31: 43-46, 1956

13) Meng H, Feng H, Le F, Lu JY: Neuroendoscopic management of symptomatic septum pellucidum cysts. Neurosurgery 59: 78-83, 2006

14) Chen LM, Zhu MX, Zhang YF, et al.: A 58-year old male with cognitive deteriorations caused by septum pellucidum cyst: A case report. Front Aging Neurosci 9: 299, 2017

Corresponding author: Naokado Ikeda, MD, PhD Department of Neurosurgery and Endovascular Neurosurgery, Osaka Medical and Pharmaceutical University, 2-7 DaigakuMachi, Takatsuki, Osaka 569-8686, Japan.

e-mail: naokado.ikeda@ompu.ac.jp 